\title{
On Stević-Sharma operator from the Zygmund space to the Bloch-Orlicz space
}

\section{Zhi-jie Jiang*}

"Correspondence: matjzj@126.com School of Science, Sichuan

University of Science and Engineering, Zigong, Sichuan 643000, P.R. China

\section{黑 Springer}

\begin{abstract}
Let $\mathbb{D}$ be the open unit disk in the complex plane $\mathbb{C}, \varphi$ an analytic self-map of $\mathbb{D}$ and $H(\mathbb{D})$ the space of all analytic functions on $\mathbb{D}$. In order to unify the products of composition, multiplication, and differentiation operators, Stević and Sharma introduced the following so-called Stević-Sharma operator:

$T_{\psi_{1}, \psi_{2}, \varphi} f(z)=\psi_{1}(z) f(\varphi(z))+\psi_{2}(z) f^{\prime}(\varphi(z)), f \in H(\mathbb{D})$, where $\psi_{1}, \psi_{2} \in H(\mathbb{D})$. Here we characterize the boundedness and compactness of the operator $T_{\psi_{1}, \psi_{2}, \varphi}$ from the Zygmund space to the Bloch-Orlicz space.
\end{abstract}

MSC: Primary 47B38; secondary 47B33; 47B37

Keywords: Zygmund space; Bloch-Orlicz space; Stević-Sharma operator; boundedness; compactness

\section{Introduction}

Let $\mathbb{D}=\{z \in \mathbb{C}:|z|<1\}$ be the open unit disk in the complex plane $\mathbb{C}$ and $H(\mathbb{D})$ the class of all analytic functions on $\mathbb{D}$. Let $\varphi$ be an analytic self-map of $\mathbb{D}$ and $\psi \in H(\mathbb{D})$. The weighted composition operator $W_{\varphi, \psi}$ on $H(\mathbb{D})$ is defined by

$$
W_{\varphi, \psi} f(z)=\psi(z) f(\varphi(z)), \quad z \in \mathbb{D} .
$$

If $\psi \equiv 1$, it becomes the composition operator, usually denoted by $C_{\varphi}$. If $\varphi(z)=z$, it becomes the multiplication operator, usually denoted by $M_{\psi}$. Hence, since $W_{\varphi, \psi}=M_{\psi} C_{\varphi}$, it is a product-type operator. A standard problem is to provide function theoretic characterizations when $\varphi$ and $\psi$ induce a bounded or compact weighted composition operator (see, e.g., $[1-5]$ and the references therein).

A systematic study of other product-type operators started by Stević et al. since the publication of papers [6] and [7]. Before that there were a few papers in the topic, e.g., [8]. The differentiation operator on $H(\mathbb{D})$ is defined by

$$
D f(z)=f^{\prime}(z), \quad z \in \mathbb{D} \text {. }
$$

The next two product-type operators $D C_{\varphi}$ and $C_{\varphi} D$, attracted some attention first (see, e.g., [9-12] and the references therein). The publication of [7] attracted some attention in product-type operators involving integral-type ones (see, e.g., [13-17] and the references therein). Since that time there has been a great interest in various product-type operators

(c) 2015 Jiang. This article is distributed under the terms of the Creative Commons Attribution 4.0 International License (http://creativecommons.org/licenses/by/4.0/), which permits unrestricted use, distribution, and reproduction in any medium, provided you give appropriate credit to the original author(s) and the source, provide a link to the Creative Commons license, and indicate if changes were made. 
on spaces of holomorphic functions. For example, the six product-type operators from Bergman spaces to Bloch type spaces

$$
M_{\psi} C_{\varphi} D, \quad M_{\psi} D C_{\varphi}, \quad C_{\varphi} M_{\psi} D, \quad C_{\varphi} D M_{\psi}, \quad D C_{\varphi} M_{\psi}, \quad D M_{\psi} C_{\varphi}
$$

were studied by Sharma in [18]. The next product-type operators $W_{\varphi, \psi} D$ and $D W_{\varphi, \psi}$, which were considered in [19] and [20], are included in (1) as the first and sixth operators, respectively. For some other product-type operators, see, e.g., [14, 21-29] and the references therein.

In order to treat operators in (1) in a unified manner, Stević and Sharma introduced the following so-called Stević-Sharma operator:

$$
T_{\psi_{1}, \psi_{2}, \varphi} f(z)=\psi_{1} f(\varphi(z))+\psi_{2}(z) f^{\prime}(\varphi(z)), \quad f \in H(\mathbb{D}) .
$$

For example, in [30] and [31] the operator was studied on the weighted Bergman space.

By using Stević-Sharma operator all six possible products of composition, multiplication, and differentiation operators can be obtained. More specifically we have

$$
\begin{aligned}
& M_{\psi} C_{\varphi} D=T_{0, \psi, \varphi}, \quad M_{\psi} D C_{\varphi}=T_{0, \psi \varphi^{\prime}, \varphi}, \quad C_{\varphi} M_{\psi} D=T_{0, \psi \circ \varphi, \varphi}, \\
& C_{\varphi} D M_{\psi}=T_{\psi^{\prime} \circ \varphi, \psi \circ \varphi, \varphi}, \quad D M_{\psi} C_{\varphi}=T_{\psi^{\prime}, \psi \varphi^{\prime}, \varphi}, \quad D C_{\varphi} M_{\psi}=T_{\varphi^{\prime} \psi^{\prime} \circ \varphi, \varphi^{\prime} \psi \circ \varphi, \varphi} .
\end{aligned}
$$

Furthermore, by using this operator all possible difference operators of product-type operators in (1) can also be obtained. For example

$$
\begin{aligned}
& M_{\psi_{1}} C_{\varphi} D-M_{\psi_{2}} D C_{\varphi}=T_{0, \psi_{1}-\psi_{2} \varphi^{\prime}, \varphi}, \quad M_{\psi_{1}} C_{\varphi} D-C_{\varphi} M_{\psi_{2}} D=T_{0, \psi_{1}-\psi_{2} \circ \varphi, \varphi}, \\
& M_{\psi_{1}} C_{\varphi} D-C_{\varphi} D M_{\psi_{2}}=T_{-\psi_{2}^{\prime} \circ \varphi, \psi_{1}-\psi_{2} \circ \varphi, \varphi}, \quad M_{\psi_{1}} C_{\varphi} D-D M_{\psi_{2}} C_{\varphi}=T_{-\psi_{2}^{\prime}, \psi_{1}-\psi_{2} \varphi^{\prime}, \varphi}, \\
& M_{\psi_{1}} C_{\varphi} D-D C_{\varphi} M_{\psi_{2}}=T_{-\varphi^{\prime} \psi_{2}^{\prime} \circ \varphi, \psi_{1}-\varphi^{\prime} \psi_{2} \circ \varphi, \varphi}, \quad M_{\psi_{1}} D C_{\varphi}-C_{\varphi} M_{\psi_{2}} D=T_{0, \psi_{1} \varphi^{\prime}-\psi_{2} \circ \varphi, \varphi}, \\
& M_{\psi_{1}} D C_{\varphi}-C_{\varphi} D M_{\psi_{2}}=T_{-\psi_{2}^{\prime} \circ \varphi, \psi_{1} \varphi^{\prime}-\psi_{2} \circ \varphi, \varphi}, \quad M_{\psi_{1}} D C_{\varphi}-D M_{\psi_{2}} C_{\varphi}=T_{-\psi_{2}^{\prime},\left(\psi_{1}-\psi_{2}\right) \varphi^{\prime}, \varphi}, \\
& M_{\psi_{1}} D C_{\varphi}-D C_{\varphi} M_{\psi_{2}}=T_{-\varphi^{\prime} \psi_{2}^{\prime} \circ \varphi, \psi_{1} \varphi^{\prime}-\varphi^{\prime} \psi_{2} \circ \varphi, \varphi}, \\
& C_{\varphi} M_{\psi_{1}} D-C_{\varphi} D M_{\psi_{2}}=T_{-\psi_{2}^{\prime} \circ \varphi,\left(\psi_{1}-\psi_{2}\right) \circ \varphi, \varphi} \\
& C_{\varphi} M_{\psi_{1}} D-D M_{\psi_{2}} C_{\varphi}=T_{-\psi_{2}^{\prime}, \psi_{1} \varphi-\psi_{2} \varphi^{\prime}, \varphi}, \\
& C_{\varphi} M_{\psi_{1}} D-D C_{\varphi} M_{\psi_{2}}=T_{-\varphi^{\prime} \psi_{2}^{\prime} \circ \varphi, \psi_{1} \circ \varphi-\varphi^{\prime} \psi_{2} \circ \varphi, \varphi} \\
& C_{\varphi} D M_{\psi_{1}}-D M_{\psi_{2}} C_{\varphi}=T_{\psi_{1}^{\prime} \circ \varphi-\psi_{2}^{\prime}, \psi_{1} \circ \varphi-\psi_{2} \varphi, \varphi} \\
& C_{\varphi} D M_{\psi_{1}}-D C_{\varphi} M_{\psi_{2}}=T_{\psi_{1}^{\prime} \circ \varphi-\varphi^{\prime} \psi_{2} \circ \varphi, \psi_{1} \circ \varphi-\varphi^{\prime} \psi_{2} \circ \varphi, \varphi} \\
& D M_{\psi_{1}} C_{\varphi}-D C_{\varphi} M_{\psi_{2}}=T_{\psi_{1}^{\prime}-\varphi^{\prime} \psi_{2} \circ \varphi, \psi_{1} \varphi^{\prime}-\varphi^{\prime} \psi_{2} \circ \varphi, \varphi}
\end{aligned}
$$

etc., where $\psi_{1}, \psi_{2} \in H(\mathbb{D})$. In this paper we characterize the boundedness and compactness of the Stević-Sharma operator from the Zygmund space to the Bloch-Orlicz space. As the applications of our main results, readers can obtain some characterizations for the boundedness and compactness for all six product-type operators in (1), as well as above mentioned differences operators from the Zygmund space to the Bloch-Orlicz space. 
Now we present the needed spaces and some facts. For $\alpha>0$, the weighted Zygmund space $\mathcal{Z}_{\alpha}$ consists of all $f \in H(\mathbb{D})$ such that

$$
\sup _{z \in \mathbb{D}}\left(1-|z|^{2}\right)^{\alpha}\left|f^{\prime \prime}(z)\right|<\infty
$$

It is a Banach space with the norm

$$
\|f\|_{\mathcal{Z}_{\alpha}}=|f(0)|+\left|f^{\prime}(0)\right|+\sup _{z \in \mathbb{D}}\left(1-|z|^{2}\right)^{\alpha}\left|f^{\prime \prime}(z)\right|
$$

When $\alpha=1$, this space is the Zygmund space and is denoted by $\mathcal{Z}$ [32]. From Zygmund's theorem (see Theorem 5.3 in [33]), we know that $f \in \mathcal{Z}$ if and only if $f$ is continuous on $\overline{\mathbb{D}}$ and

$$
\sup _{h>0, \theta \in \mathbb{R}} \frac{\left|f\left(e^{i(\theta+h)}\right)+f\left(e^{i(\theta-h)}\right)-2 f\left(e^{i \theta}\right)\right|}{h}<\infty .
$$

For some results on Zygmund-type spaces and some concrete operators on them, see, for example, $[15,23,32]$ and the references therein.

Recently, the Bloch-Orlicz space was introduced in [4] by Ramos Fernández. More precisely, let $\Psi$ be a strictly increasing convex function such that $\Psi(0)=0$. From these conditions it follows that $\lim _{t \rightarrow+\infty} \Psi(t)=+\infty$. The Bloch-Orlicz space associated with the function $\Psi$, denoted by $\mathcal{B}^{\Psi}$, is the class of all $f \in H(\mathbb{D})$ such that

$$
\sup _{z \in \mathbb{D}}\left(1-|z|^{2}\right) \Psi\left(\lambda\left|f^{\prime}(z)\right|\right)<\infty
$$

for some $\lambda>0$ depending on $f$. The Minkowski functional

$$
\|f\|_{\Psi}=\inf \left\{k>0: S_{\Psi}\left(\frac{f^{\prime}}{k}\right) \leq 1\right\}
$$

defines a seminorm for $\mathcal{B}^{\Psi}$, where

$$
S_{\Psi}(f)=\sup _{z \in \mathbb{D}}\left(1-|z|^{2}\right) \Psi(|f(z)|)
$$

Moreover, $\mathcal{B}^{\Psi}$ is a Banach space with the norm

$$
\|f\|_{\mathcal{B}^{\Psi}}=|f(0)|+\|f\|_{\Psi}
$$

In fact, Ramos Fernández in [4] proved that $\mathcal{B}^{\Psi}$ is isometrically equal to $\mu_{\Psi}$-Bloch space, where

$$
\mu_{\Psi}(z)=\frac{1}{\Psi^{-1}\left(\frac{1}{1-|z|^{2}}\right)}, \quad z \in \mathbb{D} .
$$

Thus, for $f \in \mathcal{B}^{\Psi}$ it follows that

$$
\|f\|_{\mathcal{B}^{\Psi}}=|f(0)|+\sup _{z \in \mathbb{D}} \mu_{\Psi}(z)\left|f^{\prime}(z)\right|
$$


This equivalent norm is useful to us for the study of operator $T_{\psi_{1}, \psi_{2}, \varphi}$ from the Zygmund space to the Bloch-Orlicz space. It is obvious to see that if $\Psi(t)=t^{p}$ with $p>0$, then the space $\mathcal{B}^{\Psi}$ coincides with the weighted Bloch space $\mathcal{B}^{\alpha}$, where $\alpha=1 / p$. Also, if $\Psi(t)=t \log (1+t)$, then $\mathcal{B}^{\Psi}$ coincides with the Log-Bloch space (see [34]). For the generalization of the Log-Bloch spaces, see, for example, [35, 36].

Let $X$ and $Y$ be Banach spaces. It is said that a linear operator $L: X \rightarrow Y$ is bounded if there exists a positive constant $K$ such that

$$
\|L f\|_{Y} \leq K\|f\|_{X}
$$

for all $f \in X$. The operator $L: X \rightarrow Y$ is said to be compact if it maps bounded sets into relatively compact sets. It is well known that the norm of operator $L: \mathcal{Z} \rightarrow \mathcal{B}^{\Psi}$ is defined by

$$
\|L\|_{\mathcal{Z} \rightarrow \mathcal{B}^{\Psi}}=\sup _{\|f\| \mathcal{Z} \leq 1}\|L f\|_{\mathcal{B}^{\Psi}}
$$

and written by $\|L\|$.

Throughout this paper, a positive constant $C$ may differ from one occurrence to the other. The notation $a \lesssim b$ means that there exists a positive constant $C$ such that $a \leq C b$. When $a \lesssim b$ and $b \lesssim a$, we write $a \simeq b$.

\section{Main results and proofs}

In order to characterize the compactness, we need the following result, which is proved in a standard way [5]. So, the proof is omitted.

Lemma 1 Let $\varphi$ be an analytic self-map of $\mathbb{D}$ and $\psi_{1}, \psi_{2} \in H(\mathbb{D})$. Then the bounded operator $T_{\psi_{1}, \psi_{2}, \varphi}: \mathcal{Z} \rightarrow \mathcal{B}^{\Psi}$ is compact if and only iffor every bounded sequence $\left\{f_{j}\right\}_{j \in \mathbb{N}}$ in $\mathcal{Z}$ such that $f_{j} \rightarrow 0$ uniformly on every compact subset of $\mathbb{D}$ as $j \rightarrow \infty$, it follows that

$$
\lim _{j \rightarrow \infty}\left\|T_{\psi_{1}, \psi_{2}, \varphi} f_{j}\right\|_{\mathcal{B}^{\Psi}}=0 .
$$

We state the following useful result whose first estimate was essentially proved in [37], while the second essentially follows from the point evaluation estimate for the Bloch functions (see, e.g., [38]). See also [2].

Lemma 2 For each $f \in \mathcal{Z}$ and $z \in \mathbb{D}$, it follows that

$$
|f(z)| \leq\|f\|_{\mathcal{Z}} \quad \text { and } \quad\left|f^{\prime}(z)\right| \leq \log \frac{e}{1-|z|^{2}}\|f\|_{\mathcal{Z}}
$$

The following lemma was proved in [37], Lemma 2.5.

Lemma 3 Let $\left\{f_{j}\right\}_{j \in \mathbb{N}}$ be a bounded sequence in $\mathcal{Z}$ which uniformly converges to zero on compact subsets of $\mathbb{D}$ as $j \rightarrow \infty$. Then

$$
\lim _{j \rightarrow \infty} \sup _{z \in \mathbb{D}}\left|f_{j}(z)\right|=0
$$


For $w \in \mathbb{D}$ and $1 / 2<|w|<1$, we define the function

$$
f_{w}(z)=\left(z-\frac{1}{\bar{w}}\right)\left[\left(1+\log \frac{e}{1-\bar{w} z}\right)^{2}+1\right]
$$

By using this function, the test functions in the Zygmund space can be obtained as follows:

$$
\begin{aligned}
& g_{w}(z)=f_{w}(z)\left(\log \frac{e}{1-|w|^{2}}\right)^{-1}, \\
& h_{w}(z)=f_{w}(z)\left(\log \frac{e}{1-|w|^{2}}\right)^{-1}-\int_{0}^{z} \log \frac{e}{1-\bar{w} \lambda} d \lambda .
\end{aligned}
$$

From [9] we have the next result on the functions $g_{w}$ and $h_{w}$.

Lemma 4 Let $w \in \mathbb{D}$ and $1 / 2<|w|<1$. Then

$$
g_{w}^{\prime}(w)=\log \frac{e}{1-|w|^{2}}, \quad g_{w}^{\prime \prime}(w)=\frac{2 \bar{w}}{1-|w|^{2}}, \quad h_{w}^{\prime \prime}(w)=\frac{\bar{w}}{1-|w|^{2}}
$$

Moreover,

$$
\sup _{1 / 2<|w|<1}\left\|g_{w}\right\|_{\mathcal{Z}} \lesssim 1, \quad \sup _{1 / 2<|w|<1}\left\|h_{w}\right\|_{\mathcal{Z}} \lesssim 1
$$

Now we characterize the boundedness of the operator $T_{\psi_{1}, \psi_{2}, \varphi}: \mathcal{Z} \rightarrow \mathcal{B}^{\Psi}$.

Theorem 1 Let $\varphi$ be an analytic self-map of $\mathbb{D}$ and $\psi_{1}, \psi_{2} \in H(\mathbb{D})$. Then the following statements are equivalent.

(i) The operator $T_{\psi_{1}, \psi_{2}, \varphi}: \mathcal{Z} \rightarrow \mathcal{B}^{\Psi}$ is bounded.

(ii) The functions $\psi_{1}, \psi_{2}$, and $\varphi$ satisfy the following conditions:

$$
\begin{aligned}
& M_{1}:=\sup _{z \in \mathbb{D}} \mu_{\Psi}(z)\left|\psi_{1}^{\prime}(z)\right|<\infty, \\
& M_{2}:=\sup _{z \in \mathbb{D}} \mu_{\Psi}(z)\left|\psi_{1}(z) \varphi^{\prime}(z)+\psi_{2}^{\prime}(z)\right| \log \frac{e}{1-|\varphi(z)|^{2}}<\infty,
\end{aligned}
$$

and

$$
M_{3}:=\sup _{z \in \mathbb{D}} \frac{\mu_{\Psi}(z)\left|\psi_{2}(z)\right|\left|\varphi^{\prime}(z)\right|}{1-|\varphi(z)|^{2}}<\infty
$$

Moreover, if the operator $T_{\psi_{1}, \psi_{2}, \varphi}: \mathcal{Z} \rightarrow \mathcal{B}^{\Psi}$ is nonzero and bounded, then

$$
\left\|T_{\psi_{1}, \psi_{2}, \varphi}\right\| \simeq 1+M_{1}+M_{2}+M_{3}
$$

Proof (i) $\Rightarrow$ (ii). Suppose that $T_{\psi_{1}, \psi_{2}, \varphi}: \mathcal{Z} \rightarrow \mathcal{B}^{\Psi}$ is bounded. For a fixed $w \in \mathbb{D}$ and $|\varphi(w)|>$ $1 / 2$, let $f(z)=h_{\varphi(w)}(z)-c_{1}+c_{2}$, where

$$
c_{1}=g_{\varphi(w)}(\varphi(w))=f_{\varphi(w)}(\varphi(w))\left(\log \frac{e}{1-|\varphi(w)|^{2}}\right)^{-1}, \quad c_{2}=\int_{0}^{\varphi(w)} \log \frac{e}{1-\overline{\varphi(w)} \lambda} d \lambda
$$


Then by Lemma 4

$$
f(\varphi(w))=f^{\prime}(\varphi(w))=0, \quad f^{\prime \prime}(\varphi(w))=h_{\varphi(w)}^{\prime \prime}(\varphi(w))=\frac{\overline{\varphi(w)}}{1-|\varphi(w)|^{2}}
$$

By using the boundedness of $T_{\psi_{1}, \psi_{2}, \varphi}: \mathcal{Z} \rightarrow \mathcal{B}^{\Psi}$ to the function $f$, we have

$$
M_{3}(w):=\frac{\mu_{\Psi}(w)|\varphi(w)|\left|\psi_{2}(w)\right|\left|\varphi^{\prime}(w)\right|}{1-|\varphi(w)|^{2}}=\mu_{\Psi}(w)\left|\left(T_{\psi_{1}, \psi_{2}, \varphi} f\right)^{\prime}(w)\right| \leq C\left\|T_{\psi_{1}, \psi_{2}, \varphi}\right\|,
$$

from which we get

$$
\sup _{|\varphi(z)|>1 / 2} M_{3}(z) \leq C\left\|T_{\psi_{1}, \psi_{2}, \varphi}\right\|
$$

From (4) it follows that

$$
\sup _{|\varphi(z)|>1 / 2} \frac{\mu_{\Psi}(z)\left|\psi_{2}(z)\right|\left|\varphi^{\prime}(z)\right|}{1-|\varphi(z)|^{2}} \leq 2 \sup _{|\varphi(z)|>1 / 2} M_{3}(z) \leq C\left\|T_{\psi_{1}, \psi_{2}, \varphi}\right\| .
$$

Let $h_{0}(z) \equiv 1 \in \mathcal{Z}$. Then by the boundedness of $T_{\psi_{1}, \psi_{2}, \varphi}: \mathcal{Z} \rightarrow \mathcal{B}^{\Psi}$, we obtain

$$
M_{1}=\sup _{z \in \mathbb{D}} \mu_{\Psi}(z)\left|\psi_{1}^{\prime}(z)\right| \leq\left\|T_{\psi_{1}, \psi_{2}, \varphi} h_{0}\right\| \leq C\left\|T_{\psi_{1}, \psi_{2}, \varphi}\right\| .
$$

Considering $h_{1}(z)=z \in \mathcal{Z}$, by the boundedness of $T_{\psi_{1}, \psi_{2}, \varphi}: \mathcal{Z} \rightarrow \mathcal{B}^{\Psi}$ we have

$$
\sup _{z \in \mathbb{D}} \mu_{\Psi}(z)\left|\psi_{1}^{\prime}(z) \varphi(z)+\psi_{1}(z) \varphi^{\prime}(z)+\psi_{2}^{\prime}(z)\right| \leq C\left\|T_{\psi_{1}, \psi_{2}, \varphi}\right\|
$$

From (6), (7), the boundedness of $\varphi$, and the triangle inequality, we obtain

$$
L_{1}:=\sup _{z \in \mathbb{D}} \mu_{\Psi}(z)\left|\psi_{1}(z) \varphi^{\prime}(z)+\psi_{2}^{\prime}(z)\right| \leq C\left\|T_{\psi_{1}, \psi_{2}, \varphi}\right\| .
$$

Considering $h_{2}(z)=z^{2} \in \mathcal{Z}$, we have

$$
\sup _{z \in \mathbb{D}} \mu_{\Psi}(z)\left|\psi_{1}^{\prime}(z)(\varphi(z))^{2}+2\left(\psi_{1}(z) \varphi^{\prime}(z)+\psi_{2}^{\prime}(z)\right) \varphi(z)+2 \psi_{2}(z) \varphi^{\prime}(z)\right| \leq C\left\|T_{\psi_{1}, \psi_{2}, \varphi}\right\|
$$

From (6), (8), (9), the boundedness of $\varphi^{2}$, and the triangle inequality, we get

$$
L_{2}:=\sup _{z \in \mathbb{D}} \mu_{\Psi}(z)\left|\psi_{2}(z)\right|\left|\varphi^{\prime}(z)\right| \leq C\left\|T_{\psi_{1}, \psi_{2}, \varphi}\right\|
$$

Then from (10) we have

$$
\sup _{|\varphi(z)| \leq 1 / 2} \frac{\mu_{\Psi}(z)\left|\psi_{2}(z) \| \varphi^{\prime}(z)\right|}{1-|\varphi(z)|^{2}} \leq C\left\|T_{\psi_{1}, \psi_{2}, \varphi}\right\| .
$$

From (5) and (11) we finally have $M_{3}<\infty$. 
Now we prove that $M_{2}<\infty$. For a fixed $w \in \mathbb{D}$ and $|\varphi(w)|>1 / 2$, let $g(z)=g_{\varphi(w)}(z)-c_{1}$. Then

$$
g(\varphi(w))=0, \quad g^{\prime}(\varphi(w))=\log \frac{e}{1-|\varphi(w)|^{2}}, \quad g^{\prime \prime}(\varphi(w))=\frac{2 \overline{\varphi(w)}}{1-|\varphi(w)|^{2}}
$$

By using the boundedness of $T_{\psi_{1}, \psi_{2}, \varphi}: \mathcal{Z} \rightarrow \mathcal{B}^{\Psi}$, we have

$$
\begin{aligned}
& \mu_{\Psi}(w)\left|\left(\psi_{1}(w) \varphi^{\prime}(w)+\psi_{2}^{\prime}(w)\right) \log \frac{e}{1-|\varphi(w)|^{2}}+2 \frac{\overline{\varphi(w)} \psi_{2}(w) \varphi^{\prime}(w)}{1-|\varphi(w)|^{2}}\right| \\
& =\mu_{\Psi}(w)\left|\left(T_{\psi_{1}, \psi_{2}, \varphi} g\right)^{\prime}(w)\right| \leq C\left\|T_{\psi_{1}, \psi_{2}, \varphi}\right\| .
\end{aligned}
$$

From (4), (12), and the triangle inequality, it follows that

$$
\begin{aligned}
\mu_{\Psi}(w)\left|\psi_{1}(w) \varphi^{\prime}(w)+\psi_{2}^{\prime}(w)\right| \log \frac{e}{1-|\varphi(w)|^{2}} & \leq 2 M_{3}(w)+C\left\|T_{\psi_{1}, \psi_{2}, \varphi}\right\| \\
& \leq C\left\|T_{\psi_{1}, \psi_{2}, \varphi}\right\|
\end{aligned}
$$

and then

$$
\sup _{|\varphi(z)|>1 / 2} \mu_{\Psi}(z)\left|\psi_{1}(z) \varphi^{\prime}(z)+\psi_{2}^{\prime}(z)\right| \log \frac{e}{1-|\varphi(z)|^{2}} \leq C\left\|T_{\psi_{1}, \psi_{2}, \varphi}\right\| .
$$

From (8), we obtain

$$
\sup _{|\varphi(z)| \leq 1 / 2} \mu_{\Psi}(z)\left|\psi_{1}(z) \varphi^{\prime}(z)+\psi_{2}^{\prime}(z)\right| \log \frac{e}{1-|\varphi(z)|^{2}} \leq L_{1} \log \frac{4 e}{3} \leq C\left\|T_{\psi_{1}, \psi_{2}, \varphi}\right\|
$$

Hence, from (14) and (15) we have $M_{2}<\infty$.

(ii) $\Rightarrow$ (i). By Lemma 2 , for all $f \in \mathcal{Z}$ we have

$$
\begin{aligned}
\mu_{\Psi}(z) & \left|\left(T_{\psi_{1}, \psi_{2}, \varphi} f\right)^{\prime}(z)\right| \\
= & \mu_{\Psi}(z)\left|\psi_{1}^{\prime}(z) f(\varphi(z))+\left(\psi_{1}(z) \varphi^{\prime}(z)+\psi_{2}^{\prime}(z)\right) f^{\prime}(\varphi(z))+\psi_{2}(z) \varphi^{\prime}(z) f^{\prime \prime}(\varphi(z))\right| \\
\leq & \mu_{\Psi}(z)\left(\left|\psi_{1}^{\prime}(z)\right||f(\varphi(z))|+\left|\psi_{1}(z) \varphi^{\prime}(z)+\psi_{2}^{\prime}(z)\right|\left|f^{\prime}(\varphi(z))\right|\right. \\
& \left.\quad+\left|\psi_{2}(z)\right|\left|\varphi^{\prime}(z)\right|\left|f^{\prime \prime}(\varphi(z))\right|\right) \\
\leq & \left(M_{1}+M_{2}+M_{3}\right)\|f\|_{\mathcal{Z}} .
\end{aligned}
$$

It is clear that

$$
\left|T_{\psi_{1}, \psi_{2}, \varphi} f(0)\right| \leq C\|f\|_{\mathcal{Z}}
$$

Hence from (16) and (17) it follows that $T_{\psi_{1}, \psi_{2}, \varphi}: \mathcal{Z} \rightarrow \mathcal{B}^{\Psi}$ is bounded.

Suppose that the operator $T_{\psi_{1}, \psi_{2}, \varphi}: \mathcal{Z} \rightarrow \mathcal{B}^{\Psi}$ is nonzero and bounded. Then from the proof of (i) $\Rightarrow$ (ii) it is not hard to see that

$$
M_{1}+M_{2}+M_{3} \lesssim\left\|T_{\psi_{1}, \psi_{2}, \varphi}\right\|
$$


Since the operator $T_{\psi_{1}, \psi_{2}, \varphi}: \mathcal{Z} \rightarrow \mathcal{B}^{\Psi}$ is nonzero, we have $\left\|T_{\psi_{1}, \psi_{2}, \varphi}\right\|>0$. From this we can find a positive constant $C$ such that $1 \leq C\left\|T_{\psi_{1}, \psi_{2}, \varphi}\right\|$, which means that

$$
1 \lesssim\left\|T_{\psi_{1}, \psi_{2}, \varphi}\right\|
$$

Then combing (18) and (19) gives

$$
1+M_{1}+M_{2}+M_{3} \lesssim\left\|T_{\psi_{1}, \psi_{2}, \varphi}\right\|
$$

It is clear from (16) and (17) that

$$
\left\|T_{\psi_{1}, \psi_{2}, \varphi}\right\| \lesssim 1+M_{1}+M_{2}+M_{3}
$$

Hence from (20) and (21) the asymptotic expression of $\left\|T_{\psi_{1}, \psi_{2}, \varphi}\right\|$ follows. The proof is finished.

Next we characterize the compactness of operator $T_{\psi_{1}, \psi_{2}, \varphi}: \mathcal{Z} \rightarrow \mathcal{B}^{\Psi}$.

Theorem 2 Let $\varphi$ be an analytic self-map of $\mathbb{D}$ and $\psi_{1}, \psi_{2} \in H(\mathbb{D})$. Then the following statements are equivalent.

(i) The operator $T_{\psi_{1}, \psi_{2}, \varphi}: \mathcal{Z} \rightarrow \mathcal{B}^{\Psi}$ is compact.

(ii) The functions $\psi_{1}, \psi_{2}$, and $\varphi$ satisfy the following conditions:

$$
\begin{aligned}
& M_{1}:=\sup _{z \in \mathbb{D}} \mu_{\Psi}(z)\left|\psi_{1}^{\prime}(z)\right|<\infty, \\
& L_{1}:=\sup _{z \in \mathbb{D}} \mu_{\Psi}(z)\left|\psi_{1}(z) \varphi^{\prime}(z)+\psi_{2}^{\prime}(z)\right|<\infty, \\
& L_{2}:=\sup _{z \in \mathbb{D}} \mu_{\Psi}(z)\left|\psi_{2}(z)\right|\left|\varphi^{\prime}(z)\right|<\infty, \\
& \lim _{|\varphi(z)| \rightarrow 1^{-}} \mu_{\Psi}(z)\left|\psi_{1}(z) \varphi^{\prime}(z)+\psi_{2}^{\prime}(z)\right| \log \frac{e}{1-|\varphi(z)|^{2}}=0,
\end{aligned}
$$

and

$$
\lim _{|\varphi(z)| \rightarrow 1^{-}} \frac{\mu_{\Psi}(z)\left|\psi_{2}(z)\right|\left|\varphi^{\prime}(z)\right|}{1-|\varphi(z)|^{2}}=0
$$

Proof (i) $\Rightarrow$ (ii). Suppose that (i) holds. Then it is clear that the operator $T_{\psi_{1}, \psi_{2}, \varphi}: \mathcal{Z} \rightarrow \mathcal{B}^{\Psi}$ is bounded. In the proof of Theorem 1, we have shown that $M_{1}<\infty, L_{1}<\infty$ and $L_{2}<\infty$. Consider a sequence $\left\{\varphi\left(z_{i}\right)\right\}_{i \in \mathbb{N}}$ in $\mathbb{D}$ such that $\left|\varphi\left(z_{i}\right)\right| \rightarrow 1^{-}$as $i \rightarrow \infty$. If such a sequence does not exist, then the last two conditions (ii) obviously hold. We may suppose, without loss of generality, that $\left|\varphi\left(z_{i}\right)\right|>1 / 2$ for all $i \in \mathbb{N}$. Using this sequence, we define the function sequence

$$
f_{i}(z)=f_{\varphi\left(z_{i}\right)}(z)\left(\log \frac{e}{1-\left|\varphi\left(z_{i}\right)\right|^{2}}\right)^{-1}-\left(\log \frac{e}{1-\left|\varphi\left(z_{i}\right)\right|^{2}}\right)^{-2} \int_{0}^{z} \log ^{3} \frac{e}{1-\overline{\varphi\left(z_{i}\right)} w} d w
$$


Then from a calculation we see that $\sup _{i \in \mathbb{N}}\left\|f_{i}\right\|_{\mathcal{Z}} \leq C$ and $f_{i} \rightarrow 0$ uniformly on every compact subset of $\mathbb{D}$ as $i \rightarrow \infty$. So by Lemma 1

$$
\lim _{i \rightarrow \infty}\left\|T_{\psi_{1}, \psi_{2}, \varphi} f_{i}\right\|_{\mathcal{B}^{\Psi}}=0
$$

Moreover, we have

$$
f_{i}^{\prime}\left(\varphi\left(z_{i}\right)\right)=0, \quad f_{i}^{\prime \prime}\left(\varphi\left(z_{i}\right)\right)=-\frac{\overline{\varphi\left(z_{i}\right)}}{1-\left|\varphi\left(z_{i}\right)\right|^{2}}
$$

Hence we get

$$
\left|\frac{\mu_{\Psi}\left(z_{i}\right)\left|\psi_{2}\left(z_{i}\right)\right|\left|\varphi^{\prime}\left(z_{i}\right)\right|\left|\varphi\left(z_{i}\right)\right|}{1-\left|\varphi\left(z_{i}\right)\right|^{2}}-\mu_{\Psi}\left(z_{i}\right)\right| \psi_{1}^{\prime}\left(z_{i}\right)|| f_{i}\left(\varphi\left(z_{i}\right)\right)|| \leq\left\|T_{\psi_{1}, \psi_{2}, \varphi} f_{i}\right\|_{\mathcal{B}^{\Psi}}
$$

From this, Lemmas 1 and 3 , and since $M_{1}$ is finite, we obtain

$$
\lim _{i \rightarrow \infty} \frac{\mu_{\Psi}\left(z_{i}\right)\left|\psi_{2}\left(z_{i}\right)\right|\left|\varphi^{\prime}\left(z_{i}\right)\right|}{1-\left|\varphi\left(z_{i}\right)\right|^{2}}=0
$$

On the other hand, take the sequence $g_{i}(z)=g_{\varphi\left(z_{i}\right)}(z)-c_{i}, i \in \mathbb{N}$, where $c_{i}=g_{\varphi\left(z_{i}\right)}\left(\varphi\left(z_{i}\right)\right)$. Then $\sup _{i \in \mathbb{N}}\left\|g_{i}\right\|_{\mathcal{Z}} \leq C$,

$$
g_{i}\left(\varphi\left(z_{i}\right)\right)=0, \quad g_{i}^{\prime}\left(\varphi\left(z_{i}\right)\right)=\log \frac{e}{1-\left|\varphi\left(z_{i}\right)\right|^{2}}, \quad g_{i}^{\prime \prime}\left(z_{i}\right)=\frac{2 \overline{\varphi\left(z_{i}\right)}}{1-\left|\varphi\left(z_{i}\right)\right|^{2}}
$$

Hence we have

$$
\mu_{\Psi}\left(z_{i}\right)\left|\left(\psi_{1}\left(z_{i}\right) \varphi^{\prime}\left(z_{i}\right)+\psi_{2}^{\prime}\left(z_{i}\right)\right) \log \frac{e}{1-\left|\varphi\left(z_{i}\right)\right|^{2}}+\frac{2 \overline{\varphi\left(z_{i}\right)}}{1-\left|\varphi\left(z_{i}\right)\right|^{2}}\right| \leq\left\|T_{\psi_{1}, \psi_{2}, \varphi} g_{i}\right\|_{\mathcal{B}^{\Psi}} .
$$

By the compactness $T_{\psi_{1}, \psi_{2}, \varphi}: \mathcal{Z} \rightarrow \mathcal{B}^{\Psi}$, Lemma 1 and (22), we get

$$
\lim _{i \rightarrow \infty} \mu_{\Psi}\left(z_{i}\right)\left|\psi_{1}\left(z_{i}\right) \varphi^{\prime}\left(z_{i}\right)+\psi_{2}^{\prime}\left(z_{i}\right)\right| \log \frac{e}{1-\left|\varphi\left(z_{i}\right)\right|^{2}}=0 .
$$

(ii) $\Rightarrow$ (i). We first prove that $T_{\psi_{1}, \psi_{2}, \varphi}: \mathcal{Z} \rightarrow \mathcal{B}^{\Psi}$ is bounded. We observe that the conditions in (ii) imply that for every $\varepsilon>0$, there is an $\eta \in(0,1)$, such that for any $z \in K=\{z \in$ $\mathbb{D}:|\varphi(z)|>\eta\}$

$$
R_{1}(z):=\mu_{\Psi}(z)\left|\psi_{1}(z) \varphi^{\prime}(z)+\psi_{2}^{\prime}(z)\right| \log \frac{e}{1-|\varphi(z)|^{2}}<\varepsilon
$$

and

$$
R_{2}(z):=\frac{\mu_{\Psi}(z)\left|\psi_{2}(z)\right|\left|\varphi^{\prime}(z)\right|}{1-|\varphi(z)|^{2}}<\varepsilon
$$

From the fact $L_{1}<\infty$ and (23), we obtain

$$
M_{2}=\sup _{z \in \mathbb{D}} \mu_{\Psi}(z)\left|\psi_{1}(z) \varphi^{\prime}(z)+\psi_{2}^{\prime}(z)\right| \log \frac{e}{1-|\varphi(z)|^{2}} \leq \varepsilon+L_{1} \log \frac{e}{1-\eta^{2}} .
$$


From the fact $L_{2}<\infty$ and (24), we also obtain

$$
M_{3}=\sup _{z \in \mathbb{D}} \frac{\mu_{\Psi}(z)\left|\psi_{2}(z)\right|\left|\varphi^{\prime}(z)\right|}{1-|\varphi(z)|^{2}} \leq \varepsilon+\frac{L_{2}}{1-\eta^{2}}
$$

Hence from Theorem 1 it follows that the operator $T_{\psi_{1}, \psi_{2}, \varphi}: \mathcal{Z} \rightarrow \mathcal{B}^{\Psi}$ is bounded.

In order to prove that the operator $T_{\psi_{1}, \psi_{2}, \varphi}: \mathcal{Z} \rightarrow \mathcal{B}^{\Psi}$ is compact, by Lemma 1 we just need to prove that, if $\left\{f_{i}\right\}_{i \in \mathbb{N}}$ is a sequence in $\mathcal{Z}$ such that $\sup _{i \in \mathbb{N}}\left\|f_{i}\right\|_{\mathcal{Z}} \leq M$ and $f_{i} \rightarrow 0$ uniformly on any compact subset of $\mathbb{D}$ as $i \rightarrow \infty$, then

$$
\lim _{i \rightarrow \infty}\left\|T_{\psi_{1}, \psi_{2}, \varphi} f_{i}\right\|_{\mathcal{B}^{\Psi}}=0
$$

For such a chosen $\varepsilon$ and $\eta$, by using (23), (24), and Lemma 2 we have

$$
\begin{aligned}
\mu_{\Psi}(z) & \left|\left(T_{\psi_{1}, \psi_{2}, \varphi} f_{i}\right)^{\prime}(z)\right| \\
= & \mu_{\Psi}(z)\left|\psi_{1}^{\prime}(z) f_{i}(\varphi(z))+\left(\psi_{1}(z) \varphi^{\prime}(z)+\psi_{2}^{\prime}(z)\right) f_{i}^{\prime}(\varphi(z))+\varphi^{\prime}(z) \psi_{2}(z) f_{i}^{\prime \prime}(\varphi(z))\right| \\
\leq & \mu_{\Psi}(z)\left(\left|\psi_{1}^{\prime}(z)\right|\left|f_{i}(\varphi(z))\right|+\left|\psi_{1}(z) \varphi^{\prime}(z)+\psi_{2}^{\prime}(z)\right|\left|f_{i}^{\prime}(\varphi(z))\right|\right. \\
& \left.+\left|\varphi^{\prime}(z)\right|\left|\psi_{2}(z)\right|\left|f_{i}^{\prime \prime}(\varphi(z))\right|\right) \\
\leq & M_{1} \sup _{z \in \mathbb{D}}\left|f_{i}(z)\right|+\left(\sup _{z \in K}+\sup _{z \in \mathbb{D} \backslash K}\right) \mu_{\Psi}(z)\left|\psi_{1}(z) \varphi^{\prime}(z)+\psi_{2}^{\prime}(z)\right|\left|f_{i}^{\prime}(\varphi(z))\right| \\
& +\left(\sup _{z \in K}+\sup _{z \in \mathbb{D} \backslash K}\right) \mu_{\Psi}(z)\left|\varphi^{\prime}(z)\right|\left|\psi_{2}(z)\right|\left|f_{i}^{\prime \prime}(\varphi(z))\right| \\
\leq & 2 \varepsilon+M_{1} \sup _{z \in \mathbb{D}}\left|f_{i}(z)\right|+L_{1} \sup _{|z| \leq \eta}\left|f_{i}^{\prime}(z)\right|+L_{2} \sup _{|z| \leq \eta}\left|f_{i}^{\prime \prime}(z)\right| .
\end{aligned}
$$

Since $f_{i} \rightarrow$ uniformly on compact subsets of $\mathbb{D}$ as $i \rightarrow \infty$ implies that for each $k \in \mathbb{N}$, $f_{i}^{(k)} \rightarrow 0$ uniformly on compact subsets of $\mathbb{D}$ as $i \rightarrow \infty$, from (25) and Lemma 3 we get

$$
\lim _{i \rightarrow \infty} \sup _{z \in \mathbb{D}} \mu_{\Psi}(z)\left|\left(T_{\psi_{1}, \psi_{2}, \varphi} f_{i}\right)^{\prime}(z)\right|=0 .
$$

It is clear that

$$
\lim _{i \rightarrow \infty}\left|T_{\psi_{1}, \psi_{2}, \varphi} f_{i}(0)\right|=0
$$

From (25) and (26) we obtain

$$
\lim _{i \rightarrow \infty}\left\|T_{\psi_{1}, \psi_{2}, \varphi} f_{i}\right\|_{\mathcal{B}^{\Psi}}=0
$$

Hence from (27) and Lemma 1 , we see that $T_{\psi_{1}, \psi_{2}, \varphi}: \mathcal{Z} \rightarrow \mathcal{B}^{\Psi}$ is compact. The proof is finished. 


\section{Acknowledgements}

The author would like to thank the anonymous referee very much for providing valuable suggestions for the improvement of this paper. This work was supported by the National Natural Science Foundation of China (No.11201323), the Key Fund Project of Sichuan Provincial Department of Education (No. 15ZA0221), the Sichuan Province University Key Laboratory of Bridge Non-destruction Detecting and Engineering Computing (No. 2013QZJ01) and the Cultivation Project of Sichuan University of Science and Engineering (No. 2015PY04).

Received: 26 June 2015 Accepted: 6 July 2015 Published online: 22 July 2015

\section{References}

1. Colonna, F, Li, S: Weighted composition operators from the minimal Möbius invariant space into the Bloch space. Mediterr. J. Math. 10(1), 395-409 (2013)

2. Esmaeili, $\mathrm{K}$, Lindström, M: Weighted composition operators between Zygmund type spaces and their essential norms. Integral Equ. Oper. Theory 75, 473-490 (2013)

3. Madigan, K, Matheson, A: Compact composition operators on the Bloch space. Trans. Am. Math. Soc. 347, $2679-2687$ (1995)

4. Ramos Fernández, JC: Composition operators on Bloch-Orlicz type spaces. Appl. Math. Comput. 217, $3392-3402$ (2010)

5. Schwartz, HJ: Composition operators on $H^{P}$. Thesis, University of Toledo (1969)

6. Li, S, Stević, S: Composition followed by differentiation between Bloch type spaces. J. Comput. Anal. Appl. 9(2), 195-205 (2007)

7. Li, S, Stević, S: Products of composition and integral type operators from $H^{\infty}$ to the Bloch space. Complex Var. Elliptic Equ. 53(5), 463-474 (2008)

8. Hibschweiler, RA, Portnoy, N: Composition followed by differentiation between Bergman and Hardy spaces. Rocky Mt. J. Math. 35(3), 843-855 (2005)

9. Li, S, Stević, S: Products of composition and differentiation operators from Zygmund spaces to Bloch spaces and Bers spaces. Appl. Math. Comput. 217, 3144-3154 (2010)

10. Ohno, S: Products of composition and differentiation on Bloch spaces. Bull. Korean Math. Soc. 46(6), $1135-1140$ (2009)

11. Stević, S: Norm and essential norm of composition followed by differentiation from $\alpha$-Bloch spaces to $H_{\mu}^{\infty}$. Appl. Math. Comput. 207, 225-229 (2009)

12. Stević, S: Products of composition and differentiation operators on the weighted Bergman space. Bull. Belg. Math. Soc. Simon Stevin 16, 623-635 (2009)

13. Krantz, S, Stević, S: On the iterated logarithmic Bloch space on the unit ball. Nonlinear Anal. TMA 71, 1772-1795 (2009)

14. Stević, S: Products of integral-type operators and composition operators from the mixed norm space to Bloch-type spaces. Sib. Math. J. 50(4), 726-736 (2009)

15. Stević, S: On an integral operator from the Zygmund space to the Bloch-type space on the unit ball. Glasg. Math. J. 51, 275-287 (2009)

16. Stević, S: On an integral-type operator from logarithmic Bloch-type and mixed-norm spaces to Bloch-type spaces Nonlinear Anal. TMA 71, 6323-6342 (2009)

17. Stević, S, Ueki, SI: Integral-type operators acting between weighted-type spaces on the unit ball. Appl. Math. Comput. 215, 2464-2471 (2009)

18. Sharma, AK: Products of composition multiplication and differentiation between Bergman and Bloch type spaces Turk. J. Math. 35, 275-291 (2011)

19. Jiang, ZJ: On a class of operators from weighted Bergman spaces to some spaces of analytic functions. Taiwan. J. Math. 15(5), 2095-2121 (2011)

20. Jiang, ZJ: On a product-type operator from weighted Bergman-Orlicz space to some weighted type spaces. Appl. Math. Comput. 256, 37-51 (2015)

21. Liu, $Y, Y u, Y$ : Products of composition, multiplication and radial derivative operators from logarithmic Bloch spaces to weighted-type spaces on the unit ball. J. Math. Anal. Appl. 423(1), 76-93 (2015)

22. Li, H, Guo, Z: On a product-type operator from Zygmund-type spaces to Bloch-Orlicz spaces. J. Math. Inequal. 2015, Article ID $132(2015)$

23. Li, S, Stević, S: Generalized composition operators on Zygmund spaces and Bloch type spaces. J. Math. Anal. Appl. 338, 1282-1295 (2008)

24. Pan, C: Generalized composition operators from $\mu$-Bloch spaces into mixed norm spaces. Ars Comb. 102, 263-268 (2011)

25. Ren, Y: On an integral-type operator from mixed norm spaces to Zygmund-type spaces. Bull. Math. Anal. Appl. 4(3), 71-77 (2012)

26. Sehba, B, Stević, S: On some product-type operators from Hardy-Orlicz and Bergman-Orlicz spaces to weighted-type spaces. Appl. Math. Comput. 233, 565-581 (2014)

27. Yang, W, Zhu, X: Generalized weighted composition operators from area Nevanlinna spaces to Bloch-type spaces. Taiwan. J. Math. 16(3), 869-883 (2012)

28. Zhu, X: Generalized weighted composition operators from Bloch spaces into Bers-type spaces. Filomat 26, 1163-1169 (2012)

29. Zhu, X: Generalized weighted composition operators on Bloch-type spaces. J. Inequal. Appl. 2015, Article ID 59 (2015). doi:10.1186/s13660-015-0580-0

30. Stević, S, Sharma, AK, Bhat, A: Products of multiplication composition and differentiation operators on weighted Bergman spaces. Appl. Math. Comput. 217, 8115-8125 (2011)

31. Stević, S, Sharma, AK, Bhat, A: Essential norm of multiplication composition and differentiation operators on weighted Bergman spaces. Appl. Math. Comput. 218, 2386-2397 (2011)

32. Li, S, Stević, S: Volterra type operators on Zygmund space. J. Inequal. Appl. 2007, Article ID 32124 (2007)

33. Duren, PL: Theory of HP Spaces. Academic Press, New York (1970) 
34. Attele, K: Toeplitz and Hankel operators on Bergman spaces. Hokkaido Math. J. 21, 279-293 (1992)

35. Stević, S: On new Bloch-type spaces. Appl. Math. Comput. 215, 841-849 (2009)

36. Stević, S: On an integral-type operator from logarithmic Bloch-type spaces to mixed-norm spaces on the unit ball. Appl. Math. Comput. 215, 3817-3823 (2010)

37. Stević, S: On an integral-type operator from Zygmund-type spaces to mixed-norm spaces on the unit ball. Abstr. Appl. Anal. 2010, Article ID 198608 (2010)

38. Stević, S: On an integral operator on the unit ball in $\mathbb{C}^{n}$. J. Inequal. Appl. 2005(1), 81-88 (2005)

Submit your manuscript to a SpringerOpen ${ }^{\circ}$ journal and benefit from:

- Convenient online submission

Rigorous peer review

- Immediate publication on acceptance

- Open access: articles freely available online

- High visibility within the field

- Retaining the copyright to your article 